\title{
ADAPTACIÓN TRANSCULTURAL PARA CHILE DEL NURSING ACTIVITIES SCORE PARA MEDIR LA CARGA DE TRABAJO DE ENFERMERÍA EN UNIDADES DE CUIDADOS INTENSIVOS
}

\section{CROSS-CULTURAL ADAPTATION FOR CHILE OF THE NURSING ACTIVITIES SCORE TO MEASURE NURSING WORKLOAD IN INTENSIVE CARE UNITS}

\author{
Katiuska Lidice Reynaldos Grandon * \\ Maria Cecilia Toffoletto* \\ Yerko Molina Muñoz ${ }^{* * *}$ \\ Katia Grillo Padilha ${ }^{* * * *}$ \\ Nannett Andrea SÁnchez Aguiera ***** \\ Elisa Isabel Maldonado Mardones ${ }^{* * * * *}$
}

\begin{abstract}
RESUMEN
Objetivo: Describir la adaptación del Nursing Activities Score al español hablado en Chile, para su utilización en unidades de cuidados intensivos en Chile. Material y métodos: Adaptación transcultural con equivalencia semántica e idiomática cuyas etapas metodológicas fueron: traducción y retrotraducción, evaluación de la validez de contenido y adaptación lingüística a la población a evaluar. Posterior a la traducción, la primera versión fue sometida a evaluación de contenido por cinco jueces expertos y se utilizó el coeficiente de validez de contenido. Para la adaptación lingüística, se aplicó metodología cualitativa por medio de un grupo focal conformado por ocho enfermeros clínicos quienes evaluaron la comprensión, viabilidad, claridad y la terminología empleada en cada ítem hasta llegar a un consenso respecto de su claridad verbal, redacción y comprensión, generando una segunda versión del instrumento. Resultados: La validez de contenido y el índice de Lyn fueron entre 0,6 y 1,0 y entre 80 y 100, respectivamente. En el grupo focal, algunas expresiones fueron ajustadas para la cultura chilena. No hubo alteraciones en el contenido del instrumento y se consideró la segunda versión como la final. Conclusiones: La adaptación transcultural de los ítems del Nursing Activities Score fue considerada bien diseñada y acorde para medir la carga de trabajo en enfermería en UCI en Chile.
\end{abstract}

Palabras clave: Carga de Trabajo; Enfermería; Adaptación Transcultural; Unidades de Cuidados Intensivos.

\footnotetext{
* Enfermera-matrona. Doctor en Ciencias Empresariales. Profesor asociado de la Facultad de Enfermería Universidad Andrés Bello. Santiago, Chile. Email: katiuska.reynaldos@unab.cl

${ }^{* *}$ Enfermera. Doctor en Enfermería. Profesor asociado de la Facultad de Enfermería Universidad Andrés Bello. Santiago, Chile. Email: mtoffoletto@inacap.cl

${ }^{* * *}$ Psicólogo. Máster en psicología de la salud y epidemiología. Profesor de la Facultad de Psicología Universidad Adolfo Ibáñez. Santiago, Chile. Email: yerko.molina@uai.cl

${ }^{* * * *}$ Enfermera. Doctor en Enfermería. Profesor titular de la Escola de Enfermagem Universidade de São Paulo. São Paulo, Brasil.Email: kgpadilh@usp.br

E**** Enfermera. Magíster en Enfermería. Coordinadora de servicios clínicos y diagnóstico. Clínica Indisa. Santiago, Chile. Email: nannet.sanchez@indisa.cl

${ }^{* * * * * *}$ Enfermera. Master in Business and Administration (). Enfermera Jefe UPC. Hospital del Trabajador-Asociación Chilena de Seguridad. Santiago, Chile.Email: emaldonado@achs.cl
} 


\section{ABSTRACT}

Objective: To describe the adaptation of the Nursing Activities Score (NAS) into Chilean Spanish for use in Chilean intensive care units. Material and methods: Cross-cultural adaptation with semantic and idiomatic equivalence. The methodological steps consisted of translation and back translation, evaluation of content validity and linguistic adaptation to the target population. After the translation, the first version was assessed for content by five expert judges using the content validity coefficient. For the linguistic adaptation, qualitative methodology was applied conducting a focus group with eight clinical nurses, who evaluated the comprehension, viability, clarity and terminology of each NAS item until agreeing upon criteria for a second version of the instrument. Results: Content validity and Lin's index values were between 0.6 and 1.0 and between 80 and 100, respectively. In the focus group conducted with the clinical nurses some expressions were adjusted to the Chilean culture. There were no changes in the content of the instrument and the second version was considered as the final one. Conclusion: The cross-cultural adaptation of the NAS items were deemed valid and reliable for measuring the workload of nursing in Chilean intensive care units.

Key words: Workload; Nursing; Cross-cultural Adaptation; Intensive Care Units.

Fecha recepción: 20/11/17 Fecha aceptación: 11/09/18

\section{INTRODUCCIÓN}

El cuidado al paciente grave, brindado en una Unidad de Cuidados Intensivos (UCI), exige la utilización de alta tecnología, realización de diversas y complicadas intervenciones terapéuticas, además del soporte constante a las familias, actividades todas que, por su complejidad, a diferencia de otros servicios, tiene una alta demanda de recursos humanos en enfermería.

La presencia de indicadores que permitan medir la cantidad de trabajo del recurso humano de enfermería, a partir de las necesidades de cuidados de los pacientes, es, en la actualidad, uno de los requisitos que garantizarían la optimización de la relación costo-beneficio y la calidad de la asistencia a la salud, debido al alto costo y a la alta demanda de recursos humanos en cantidad y calidad que requieren las $\mathrm{UCI}^{(1)}$.

La evidencia científica reporta diversos instrumentos que miden la carga de trabajo de enfermería en los servicios de UCI, siendo el instrumento Therapeutic Intervention Scoring System (TISS-28) lo más utilizado y reconocido mundialmente en el contexto de las unidades de cuidados intensivos $^{(2)}$. Fue creado con el objetivo de medir la gravedad de los pacientes y calcular la carga de trabajo de enfermería en UCI. Tras dos modificaciones, la versión es actualmente utilizada para la dotación de recursos humanos de enfermería. No obstante, se evidenció que la demanda de trabajo de enfermería en base a la gravedad del paciente, medido por el TISS-28, solo contemplaba el $34,3 \%$ de las actividades de enfermería $^{(3)}$. De esta forma, en base al TISS-28, en el año 2003, se propuso el instrumento Nursing Activities Score (NAS), el cual contempla 80,8\% de la carga de trabajo de enfermería en comparación al bajo valor exhibido por el TISS-28(4).

El NAS está conformado por un total de 23 actividades y su gran diferencia se destaca en la categoría "Actividades básicas", en la cual se contemplan las actividades de: monitoreo y controles, laboratorio, medicamentos, procedimientos de aseo, cuidados con drenajes, movilización y posicionamiento del paciente, soporte a los familiares y tareas de gestión. En el instrumento, las actividades son registradas por los enfermeros, en base a los cuidados prestados en las últimas 24 horas y cada una de las 23 actividades posee un puntaje. La suma de los puntos representa, en porcentaje, el gasto del tiempo que el paciente necesita del $100 \%$ del tiempo de trabajo del personal de enfermería ${ }^{(4)}$.

Respecto a la utilización del NAS, las evidencias demuestran la efectividad de este instrumento en la medida de la carga de trabajo de enfer- 
mería en las UCI para dimensionar el personal, evaluar costos, además de su utilización en las diversas especialidades ${ }^{(5-13)}$.

Un factor limitante de la utilización del NAS se refiere al idioma inglés en el cual fue originalmente creado. En América Latina, el instrumento se encuentra adaptado y validado solamente en Brasil $^{(1)}$ y respecto a los países de habla hispana, solamente en España ${ }^{(14)}$.

Considerando los atributos del instrumento y el interés de disponer del NAS en Chile, se propuso la adaptación transcultural, con vistas a ofrecer un instrumento innovador para determinar la dotación de recursos humanos de enfermería en los servicios críticos, como una herramienta de gestión operativamente efectiva. Según lo anterior, este estudio tuvo por objetivo describir la adaptación del Nursing Activities Score (NAS) al idioma español para su utilización en unidades de cuidados intensivos en Chile.

\section{MATERIAL Y MÉTODO}

Se trató de una adaptación transcultural con enfoque en la equivalencia semántica e idiomática, cuyas etapas metodológicas fueron: traducción y retrotraducción, evaluación de la validez de contenido y adaptación lingüística a la población a evaluar $^{(15,16)}$.

La traducción de la versión original (VO) para el idioma español fue realizada por dos traductores profesionales bilingües independientes, cuya lengua materna era el español. Uno de los traductores (T1) conocía los objetivos y los conceptos del instrumento y tenía experiencia previa en la traducción técnica de textos. El segundo traductor (T2) no tenía conocimientos previos sobre los objetivos del estudio y conceptos del instrumento. Luego de la traducción, se realizó la retrotraducción por otros dos traductores profesionales bilingües (T3 y T4) cuya lengua materna era el inglés, tenían conocimientos sobre los objetivos del estudio y trabajaron de forma independiente.

Posterior a la retrotraducción, dos investigadores y un colaborador externo realizaron una sín- tesis a partir de las 3 versiones anteriores, es decir, del instrumento original en inglés, las traducciones y retrotraducciones, para llegar a un primer informe o versión del instrumento en el idioma español (V1). Posterior a las etapas mencionadas anteriormente, el instrumento en español fue denominado Puntaje de Actividades de Enfermería. Cabe resaltar que se optó en mantener la sigla NAS y el nombre en inglés, Nursing Activities Sco$r e$, por su reconocimiento internacional.

Para llevar a cabo la evaluación de contenido del instrumento se utilizó la metodología de Lynn $^{(17)}$ que contempla la evaluación de jueces expertos de cada ítem del instrumento a validar. Se recomienda de dos a 20 jueces expertos, donde ellos catalogan los ítems del instrumento de acuerdo a su "adecuación" a lo que se pretende medir utilizando, como criterio de calidad de un ítem, que al menos un $80 \%$ de los jueces expertos lo evalúen como "adecuado". Para el presente informe se tomaron en cuenta las opiniones de 5 jueces expertos que fueron elegidos por conveniencia según los siguientes criterios de inclusión: profesionales de enfermería con más de cinco años de experiencia clínica en UCI y más de tres años de experiencia en gestión en UCI. Se utilizó un guion estructurado en el cual se les solicitó los registros referidos a la adecuación de los ítems a la variable de interés, carga de trabajo de enfermería, con los niveles "adecuado" y "no adecuado". Del mismo modo, se les solicitó el registro de la claridad de los ítems en los niveles "confuso" y "claro", expresando así la claridad conceptual y gramatical de los ítems. Se les pidió además, que en el caso de puntuar un ítem como "no adecuado" o "confuso", comentaran de manera explícita los cambios que le harían al ítem. Además, se utilizó el coeficiente de validez de contenido (CV), donde, de acuerdo a Lynn ${ }^{(17)}$, el valor mínimo debe ser establecido teniendo en cuenta el número de jueces expertos que, para este estudio, resultó ser un valor índice de 0,6.

Por último, en la etapa fue cualitativa, se conformó un grupo focal con ocho enfermeros/as clínicos de seis UCI de dos hospitales de Santiago de Chile y los criterios de inclusión aplicados para conformar el grupo fueron: ser enfermeros/ 
as clínicos, con un mínimo de tres años de experiencia en UCI y que representaran a las seis UCI. El reclutamiento de los enfermeros/as clínicos fue por azar, pues se trataba de un universo homogéneo en cuanto al desempeño de roles. La invitación fue por escrito, dos semanas antes del encuentro. Una semana y el día previo se les contactó por teléfono para asegurar su asistencia en dependencias de la Universidad. A cada uno de los participantes se les explicó los objetivos, los procedimientos de la investigación y se solicitó la firma del consentimiento informado.

Detalladamente, los profesionales de enfermería leyeron el instrumento y lo evaluaron de acuerdo a la claridad y la terminología empleada en cada ítem. Luego, se realizó una discusión grupal de cada ítem hasta llegar a un consenso en claridad verbal, redacción y comprensión de los ítems.

Cabe señalar que el investigador principal cumplió el rol de moderador, asegurando una direccionalidad en la discusión de la comprensión y viabilidad del instrumento. Junto al moderador participó un observador que ayudó en la conducción del grupo, tomó nota de las principales impresiones verbales y no verbales, y grabó la sesión.

Posterior a la transcripción, se realizaron varias lecturas hasta aprehender las observaciones relacionadas a la claridad verbal, redacción, comprensión de los ítems, propuestas de cambios y las justificaciones para las mismas, generando la segunda versión del instrumento y versión final del documento (V2).

El proyecto fue evaluado y certificado por el Comité de Ética Científico de la Facultad de Enfermería de la Universidad Andrés Bello con el protocolo de registro de emisión L1/CECENF/81.

\section{RESULTADOS}

La Tabla 1 muestra la primera versión final en español del instrumento que fue sometida a la evaluación de contenido.

En la evaluación de contenido, el CV y el ín- dice de Lynn asumieron valores entre 0,6 y 1,0 y entre 80 y 100, respectivamente (Tabla 2), concluyendo de esta forma que el instrumento era adecuado y sus ítems concordantes para la medida de la carga de trabajo en enfermería.

En el grupo focal con los profesionales de enfermería clínicos, algunas expresiones fueron ajustadas para la cultura chilena respecto a la claridad verbal, redacción y comprensión de los ítems. En el ítem relacionado al control de signos vitales por hora, el término "por hora" fue reemplazado "por horario". El término "investigaciones de laboratorio" del ítem 2 fue sustituido por "control de exámenes de laboratorio". En el ítem 3 se agregó "preparación y administración de medicamentos". El ítem 6 sufrió alteración en el término "alzamiento en equipo" por "levantamiento del paciente por el equipo". En el ítem 13 referido a la reposición intravenosa de pérdidas de líquidos en altas cantidades medido como L/ $\mathrm{m}^{2} /$ por día, independientemente del tipo de líquido administrado, se tomó la decisión de calcular el volumen solo por las $24 \mathrm{~h}$, quedando la cantidad especificada como L/día. Lo mismo con el ítem 14 sobre el control del atrio izquierdo: catéter arterial pulmonar, con o sin mediciones del rendimiento cardiaco, en donde se agregó además de las mediciones, el término "monitorización". Por último, el término "Hiperalimentación, Nutrición Parenteral por vía intravenosa" fue reemplazado por "Nutrición Parenteral Total Central" (Cuadro 1).

Además del cambio de términos de los ítems, otros temas fueron discutidos y consensuados como la presencia de otros profesionales y técnicos involucrados en el cuidado de los pacientes, la innovación tecnológica y la necesidad de actualización del instrumento. En cuanto a la presencia de otros profesionales y técnicos, los temas discutidos se relacionaron a los cuidados de las vías respiratorias por los profesionales de kinesiología y de higiene por los técnicos paramédicos.

La segunda versión y final del instrumento quedó conformada por siete grandes categorías que contemplan 23 actividades: actividades básicas (8 actividades), soporte ventilatorio (3 actividades), soporte cardiovascular (4 actividades), 
soporte renal (2 actividades), soporte neurológico ( 1 actividad), soporte metabólico (3 activida- des) e intervenciones específicas (2 actividades) (Tabla 3).

Tabla 1. Primera versión del Nursing Activities Score traducido al español.

\section{Actividades Básicas}

Puntaje

1 Monitoreo y evaluación

1a Control de los signos vitales por hora, registro y cálculo constante del equilibrio balance hídrico

Presente a pie de cama y observación continua o activa durante 2 h o más en cualquier turno por motivos de seguridad, gravedad o tratamientos como ventilación mecánica no invasiva,

$1 \mathrm{~b}$ procedimientos de destete, nerviosismo, desorientación, posición decúbito prono, procedimientos de donación, preparación y administración de líquidos o medicamentos, asistencia en procedimientos específicos

1c Presente a pie de cama y observación activa durante $4 \mathrm{~h}$ o más en cualquier turno por motivos de seguridad, gravedad o tratamientos como los mencionados anteriormente

2 Investigaciones de laboratorio, bioquímica y microbiología

3 Administración de medicamentos, excluidos los medicamentos vasoactivos

4 Procedimientos de higiene

Ejecución de procedimientos de higiene, tales como curación de heridas y catéteres intravasculares, muda de ropa, aseo del paciente, incontinencia, vómitos, quemaduras, heridas con se-

$4 \mathrm{a}$ creciones, curaciones quirúrgicas complejas con secreciones y procedimientos especiales (por ejemplo, medidas de aislamiento, procedimientos asociados a una infección cruzada, infecciones posteriores a la limpieza de la habitación, higiene del personal)

$4 \mathrm{~b}$ Ejecución de procedimientos de higiene durante $2 \mathrm{~h}$ en cualquier turno

4c Ejecución de procedimientos de higiene durante $4 \mathrm{~h}$ en cualquier turno

5 Cuidado de drenajes de todo tipo (exceptuando sondas gástricas)

Movilización y cambio de posición, incluidos procedimientos tales como: girar al paciente, mo-

6 vilización del paciente, traslado de la cama a una silla, alzamiento en equipo (p. ej. pacientes postrados, tracción, decúbito prono)

6a Realización de procedimiento(s) hasta tres veces en $24 \mathrm{~h}$

Realización de procedimiento(s) con una frecuencia mayor que 3 veces en 24 h, o con dos en-

$6 \mathrm{~b}$ fermeras, cualquier frecuencia

6c Realización de procedimiento con tres o más enfermeras, cualquier frecuencia

Apoyo y atención de familiares y del paciente, incluidos procedimientos tales como llamadas telefónicas, entrevistas, orientación; con frecuencia el apoyo y la atención de familiares o del paciente permite al personal continuar con otras actividades de enfermería (p. ej. comunicación con los pacientes durante los procedimientos de higiene, comunicación con los familiares cuando se encuentran presentes junto a la cama del paciente y observación del paciente)

Apoyo y cuidado de los familiares o del paciente que requieran una completa dedicación por al

7a menos $1 \mathrm{~h}$ durante cualquier turno, ya sea para explicar la condición clínica, asistir en el manejo del dolor o estrés, o situaciones familiares difíciles

Apoyo y cuidado de los familiares o del paciente que requieran una completa dedicación por

$7 \mathrm{~b} 3 \mathrm{~h}$ o más durante cualquier turno, en caso de fallecimiento o situaciones demandantes (por ejemplo, gran número de parientes, problemas de idioma o parientes hostiles)

8 Tareas administrativas y de gestión 
8a Ejecutar labores de rutina, como el procesamiento de datos clínicos, solicitud de exámenes, intercambio de información profesional (por ejemplo, rondas de visitas médicas)

Realización de tareas administrativas y de gestión que requieren dedicación completa durante

$8 \mathrm{~b}$ aproximadamente $2 \mathrm{~h}$ en cualquier turno tales como actividades de investigación, protocolos en uso, procedimientos de admisión y alta de pacientes

Realización de tareas administrativas y de gestión que requieren dedicación completa durante

$8 \mathrm{c}$ aproximadamente $4 \mathrm{~h}$ o más del tiempo en cualquier turno, tales como fallecimiento del paciente y procedimientos de donación de órganos, coordinación con otras disciplinas

\section{Asistencia Respiratoria}

Asistencia respiratoria: cualquier forma de ventilación mecánica o asistida con o sin presión positiva espiratoria final con o sin relajantes musculares, respiración espontánea con o sin presión positiva espiratoria final con o sin tubo endotraqueal, oxígeno complementario por cualquier método

10 Cuidado de vías respiratorias artificiales: tubo endotraqueal o cánula de traqueotomía

Tratamiento para mejorar la función pulmonar: fisioterapia respiratoria, espirometría incenti-

11 vada, terapia inhalatoria, aspiración endotraqueal

\section{Asistencia Cardiovascular}

12 Medicación vasoactiva, independiente del tipo y la dosis

Reposición intravenosa de pérdidas de líquidos en altas cantidades, Administración de líquidos

Control del atrio izquierdo: catéter arterial pulmonar, con o sin mediciones del rendimiento cardiaco

Resucitación cardiopulmonar después de un paro durante las últimas 24 h (no considerar si se aplica únicamente golpe precordial)

\section{Asistencia Renal}

16 Técnicas de hemofiltración, técnicas de diálisis

17 Medición cuantitativa de orina (por ejemplo, mediante sonda vesical)

\section{Asistencia Neurológica}

18 Medición de presión intracraneal

\section{Asistencia Metabólica}

19 Tratamiento de las complicaciones de acidosis/alcalosis metabólica

20 Hiperalimentación, Nutrición Parenteral por vía intravenosa

Alimentación enteral mediante sonda gástrica u otros medios gastrointestinales (por ejemplo,

21 yeyunostomía)

\section{Intervenciones Específicas}

Intervenciones específicas en la unidad de cuidados intensivos: intubación endotraqueal, inserción de marcapasos, cardioversión, endoscopias, cirugías de emergencia durante las últimas 24

$22 \mathrm{~h}$, lavado gástrico, Se excluyen las intervenciones rutinarias sin consecuencias directas a la condición clínica del paciente, tales como radiografías, ecografías, electrocardiogramas, curaciones o inserción de catéteres endovenosos o arteriales

23 Intervenciones específicas fuera de la unidad de cuidados intensivos: cirugías o procedimientos de diagnóstico 
Tabla 2. Evaluación de contenido (CV) e índice de Lynn de los ítems del Nursing Activities Score.

Ítems CV Índice Lynn

\section{Actividades Básicas}

1 Monitoreo y evaluación

1a

Control de los signos vitales por hora, registro y cálculo constante del equilibrio balance hídrico.

Presente a pie de cama y observación continua o activa durante $2 \mathrm{~h}$ o más en cualquier turno por motivos de seguridad, gravedad o tratamientos como ventilación mecánica no invasiva, procedimientos de destete, nerviosismo, desorientación, posición decúbito prono, procedimientos de donación, preparación y administración de líquidos o medicamentos, asistencia en procedimientos específicos.

Presente a pie de cama y observación activa durante 4 h o más en cualquier turno

1c por motivos de seguridad, gravedad o tratamientos como los mencionados anteriormente.

2 Investigaciones de laboratorio, bioquímica y microbiología.

3 Administración de medicamentos, excluidos los medicamentos vasoactivos.

4 Procedimientos de higiene

Ejecución de procedimientos de higiene, tales como curación de heridas y catéteres intravasculares, muda de ropa, aseo del paciente, incontinencia, vómitos, quemaduras, heridas con secreciones, curaciones quirúrgicas complejas con secreciones y procedimientos especiales (por ejemplo, medidas de aislamiento, procedimientos asociados a una infección cruzada, infecciones posteriores a la limpieza de la habitación, higiene del personal).

$4 \mathrm{~b} \quad$ Ejecución de procedimientos de higiene durante $2 \mathrm{~h}$ en cualquier turno.

4c Ejecución de procedimientos de higiene durante $4 \mathrm{~h}$ en cualquier turno.

5 Cuidado de drenajes de todo tipo (exceptuando sondas gástricas).

6a Realización de procedimiento(s) hasta tres veces en $24 \mathrm{~h}$.

Realización de procedimiento(s) con una frecuencia mayor que 3 veces en 24 h, o con dos enfermeras, cualquier frecuencia.

6c Realización de procedimiento con tres o más enfermeras, cualquier frecuencia.

Apoyo y cuidado de los familiares o del paciente que requieran una completa dedi-

7a cación por al menos $1 \mathrm{~h}$ durante cualquier turno, ya sea para explicar la condición clínica, asistir en el manejo del dolor o estrés, o situaciones familiares difíciles.

Apoyo y cuidado de los familiares o del paciente que requieran una completa dedicación por $3 \mathrm{~h}$ o más durante cualquier turno, en caso de fallecimiento o situaciones demandantes (por ejemplo, gran número de parientes, problemas de idioma o parientes hostiles).

Ejecutar labores de rutina, como el procesamiento de datos clínicos, solicitud de

8a exámenes, intercambio de información profesional (por ejemplo, rondas de visitas médicas).

Realización de tareas administrativas y de gestión que requieren dedicación com-

$8 \mathrm{~b}$ pleta durante aproximadamente $2 \mathrm{~h}$ en cualquier turno tales como actividades de investigación, protocolos en uso, procedimientos de admisión y alta de pacientes. 
Realización de tareas administrativas y de gestión que requieren dedicación comple-

$8 \mathrm{c}$ ta durante aproximadamente $4 \mathrm{~h}$ o más del tiempo en cualquier turno, tales como fallecimiento del paciente y procedimientos de donación de órganos, coordinación con otras disciplinas.

\section{Asistencia Respiratoria}

Asistencia respiratoria: cualquier forma de ventilación mecánica o asistida con o sin presión positiva espiratoria final con o sin relajantes musculares, respiración espontánea con o sin presión positiva espiratoria final con o sin tubo endotraqueal, oxígeno complementario por cualquier método.

Cuidado de vías respiratorias artificiales: tubo endotraqueal o cánula de traqueotomía.

Tratamiento para mejorar la función pulmonar: fisioterapia respiratoria, espirometría incentivada, terapia inhalatoria, aspiración endotraqueal.

\section{Asistencia Cardiovascular}

12 Medicación vasoactiva, independiente del tipo y la dosis.

Reposición intravenosa de pérdidas de líquidos en altas cantidades. Administración de líquidos $>3 \mathrm{~L} / \mathrm{m}^{2} /$ por día, independientemente del tipo de líquido administrado.

Control del atrio izquierdo: catéter arterial pulmonar, con o sin mediciones del rendimiento cardiaco.

Resucitación cardiopulmonar después de un paro durante las últimas 24 h (no considerar si se aplica únicamente golpe precordial).

\section{Asistencia Renal}

16 Técnicas de hemofiltración, técnicas de diálisis.

17 Medición cuantitativa de orina (por ejemplo, mediante sonda vesical).

\section{Asistencia Metabólica}

18 Medición de presión intracraneal.

19 Tratamiento de las complicaciones de acidosis/alcalosis metabólica.

Alimentación enteral mediante sonda gástrica u otros medios gastrointestinales (por ejemplo, yeyunostomía).

\section{Intervenciones Específicas}

Intervenciones específicas en la unidad de cuidados intensivos: intubación endotraqueal, inserción de marcapasos, cardioversión, endoscopias, cirugías de emergencia durante las últimas $24 \mathrm{~h}$, lavado gástrico. Se excluyen las intervenciones rutinarias sin consecuencias directas a la condición clínica del paciente, tales como radiografías, ecografías, electrocardiogramas, curaciones o inserción de catéteres endovenosos o arteriales. 
Cuadro 1. Ítems del Nursing Activities Score ajustados según la claridad verbal, redacción y comprensión para la cultura chilena.

\begin{tabular}{|c|c|}
\hline Ítems primera versión & Ítems ajustados versión final \\
\hline $\begin{array}{l}\text { 1a. Control de los signos vitales por hora, registro y } \\
\text { cálculo constante del equilibrio balance hídrico. }\end{array}$ & $\begin{array}{l}\text { 1a. Control de los signos vitales por horario, registro } \\
\text { y cálculo constante del balance hídrico. }\end{array}$ \\
\hline $\begin{array}{l}\text { 2. Investigaciones de laboratorio, bioquímica y mi- } \\
\text { crobiología. }\end{array}$ & $\begin{array}{l}\text { 2. Control de exámenes de laboratorio, bioquímica } \\
\text { y microbiología. }\end{array}$ \\
\hline $\begin{array}{l}\text { 3. Administración de medicamentos, excluidos los } \\
\text { medicamentos vasoactivos. }\end{array}$ & $\begin{array}{l}\text { 3. Preparación y administración de medicamentos, } \\
\text { excluidos los medicamentos vasoactivos. }\end{array}$ \\
\hline $\begin{array}{l}\text { 6. Movilización y cambio de posición, incluidos pro- } \\
\text { cedimientos tales como: girar al paciente, movili- } \\
\text { zación del paciente, traslado de la cama a una silla, } \\
\text { alzamiento en equipo (p. ej. pacientes postrados, } \\
\text { tracción, decúbito prono). }\end{array}$ & $\begin{array}{l}\text { 6. Movilización y cambio de posición, incluidos pro- } \\
\text { cedimientos tales como: girar al paciente, movili- } \\
\text { zación del paciente, traslado de la cama a una silla, } \\
\text { levantamiento del paciente por el equipo (p. ej. pa- } \\
\text { cientes postrados, tracción, decúbito prono). }\end{array}$ \\
\hline $\begin{array}{l}\text { 13. Reposición intravenosa de pérdidas de líquidos } \\
\text { en altas cantidades. Administración de líquidos }>3 \text { L/ } \\
\mathbf{m}^{2} / \text { por día, independientemente del tipo de líquido } \\
\text { administrado. }\end{array}$ & $\begin{array}{l}\text { 13. Reposición intravenosa de pérdidas de líquidos } \\
\text { en altas cantidades. Administración de líquidos }>3 \text { L/ } \\
\text { día, independientemente del tipo de líquido adminis- } \\
\text { trado. }\end{array}$ \\
\hline $\begin{array}{l}\text { 14. Control del atrio izquierdo: catéter arterial pul- } \\
\text { monar, con o sin mediciones del rendimiento car- } \\
\text { diaco. }\end{array}$ & $\begin{array}{l}\text { 14. Control del atrio izquierdo: catéter arterial pul- } \\
\text { monar, con o sin monitorización y medición del } \\
\text { rendimiento cardiaco. }\end{array}$ \\
\hline $\begin{array}{l}\text { 20. Hiperalimentación, Nutrición Parenteral por } \\
\text { vía intravenosa. }\end{array}$ & 20. Nu \\
\hline
\end{tabular}

\section{DISCUSIÓN}

En el proceso de adaptación transcultural del instrumento NAS, los resultados demostraron que el mismo presenta contenido adecuado para la medida de la carga de trabajo en enfermería en las UCI chilenas.

El instrumento NAS ha sido adaptado transculturalmente en varios países como Brasil( ${ }^{(1)}$, España $^{(14)}$, Portugal ${ }^{(18)}$, Italia ${ }^{(5)}$, en donde los ajustes de algunos ítems han apuntado a mejorar su comprensión y a la necesidad de adaptar la frecuencia de los turnos.

En cuanto a los ajustes de expresiones y términos, en el ítem 13, relacionado a la reposición intravenosa, se tomó la decisión de calcular el volumen solo por las $24 \mathrm{~h}$ quedando la cantidad especificada como L/día, porque los profesionales de enfermería clínicos, participantes del grupo focal, no encontraron relevancia en la medida $\mathrm{m}^{2}$, refiriendo dificultad en la lectura y comprensión pues, en Chile, el ingreso hídrico al paciente es calculado por el total de volumen administrado en las 24 horas. Esta decisión coincide con estudios latinoamericanos sobre balance hídrico como un marcador pronóstico de la evolución clínica, en donde demuestran que en el cálculo del mismo se debe considerar el ingreso total de líquidos administrados menos el total de líquidos eliminados a cada 24 horas $^{(19,20)}$.

Además, en el ítem 3 se determinó agregar el término "preparación" a la actividad administración de medicamentos, pues los profesionales de enfermería consideraron los conceptos distintos y, por lo tanto, actividades distintas. Un estudio de revisión integradora sobre errores en la preparación y administración de medicamentos, encontró que los estudios revisados destacan la preparación y administración de medicamentos como un cuidado exclusivo de enfermería y la última de las diversas fases del proceso de adminis- 
Tabla 3. Versión final del Nursing Activities Score traducido y adaptado al español en Chile.

Actividades básicas

1 Monitoreo y evaluación

1a Control de los signos vitales por horario, registro y cálculo constante del balance hídrico.

Presente a pie de cama y observación continua o activa durante $2 \mathrm{~h}$ o más en cualquier turno por motivos de seguridad, gravedad o tratamientos como ventilación mecánica no invasiva, proce-

$1 \mathrm{~b}$ dimientos de destete, nerviosismo, desorientación, posición decúbito prono, procedimientos de donación, preparación y administración de líquidos o medicamentos, asistencia en procedimientos específicos.

1c Presente a pie de cama y observación activa durante $4 \mathrm{~h}$ o más en cualquier turno por motivos de seguridad, gravedad o tratamientos como los mencionados anteriormente (1b).

Puntaje

Control de exámenes de laboratorio, bioquímica y microbiología.

3 Preparación y administración de medicamentos, excluidos los medicamentos vasoactivos.

4 Procedimientos de higiene.

Ejecución de procedimientos de higiene, tales como curación de heridas y catéteres intravasculares, muda de ropa, aseo del paciente, incontinencia, vómitos, quemaduras, heridas con secrecio-

4a nes, curaciones quirúrgicas complejas con secreciones y procedimientos especiales (por ejemplo, medidas de aislamiento, procedimientos asociados a una infección cruzada, infecciones posteriores a la limpieza de la habitación, higiene del personal).

$4 \mathrm{~b}$ Ejecución de procedimientos de higiene durante $2 \mathrm{~h}$ o más en cualquier turno.

4c Ejecución de procedimientos de higiene durante $4 \mathrm{~h}$ o más en cualquier turno.

5 Cuidado de drenajes de todo tipo (exceptuando sondas gástricas).

Movilización y cambio de posición, incluidos procedimientos tales como: girar al paciente, mo-

6 vilización del paciente, traslado de la cama a una silla, levantamiento del paciente por el equipo (p. ej. pacientes postrados, tracción, decúbito prono).

6a Realización de procedimiento(s) hasta tres veces en $24 \mathrm{~h}$.

Realización de procedimiento(s) con una frecuencia mayor que 3 veces en 24 h, o con dos enfer-

$6 \mathrm{~b}$ meras, cualquier frecuencia.

6c Realización de procedimiento con tres o más enfermeras, cualquier frecuencia.

Apoyo y atención de familiares y del paciente, incluidos procedimientos tales como llamadas telefónicas, entrevistas, orientación. Con frecuencia, el apoyo y la atención de familiares o del

7 paciente permite al personal continuar con otras actividades de enfermería (p. ej. comunicación con los pacientes durante los procedimientos de higiene, comunicación con los familiares cuando se encuentran presentes junto a la cama del paciente y observación del paciente).

Apoyo y cuidado de los familiares o del paciente que requieran una completa dedicación por al

7a menos $1 \mathrm{~h}$ durante cualquier turno, ya sea para explicar la condición clínica, asistir en el manejo del dolor o estrés, o situaciones familiares difíciles.

Apoyo y cuidado de los familiares o del paciente que requieran una completa dedicación por $3 \mathrm{~h}$

$7 \mathrm{~b}$ o más durante cualquier turno, en caso de fallecimiento o situaciones demandantes (por ejemplo, gran número de parientes, problemas de idioma o parientes hostiles).

8 Tareas administrativas y de gestión.

8a Ejecutar labores de rutina, como el procesamiento de datos clínicos, solicitud de exámenes, intercambio de información profesional (por ejemplo, rondas de visitas médicas). 
Continuación Tabla 3.

Realización de tareas administrativas y de gestión que requieren dedicación completa durante

$8 \mathrm{~b}$ aproximadamente $2 \mathrm{~h}$ en cualquier turno tales como actividades de investigación, protocolos en uso, procedimientos de admisión y alta de pacientes.

Realización de tareas administrativas y de gestión que requieren dedicación completa durante

$8 \mathrm{c}$ aproximadamente $4 \mathrm{~h}$ o más del tiempo en cualquier turno, tales como fallecimiento del paciente y procedimientos de donación de órganos, coordinación con otros profesionales.

\section{Asistencia respiratoria}

Asistencia respiratoria: cualquier forma de ventilación mecánica o asistida con o sin presión positiva espiratoria final, con o sin relajantes musculares. Respiración espontánea con o sin presión positiva espiratoria final, con o sin tubo endotraqueal. Oxígeno complementario por cualquier método.

10 Cuidado de vías respiratorias artificiales: tubo endotraqueal o cánula de traqueotomía.

Tratamiento para mejorar la función pulmonar: fisioterapia respiratoria, espirometría incentiva-

11 da, terapia inhalatoria, aspiración endotraqueal.

\section{Asistencia cardiovascular}

12 Medicación vasoactiva, independiente del tipo y la dosis.

Reposición intravenosa de pérdidas de líquidos en altas cantidades. Administración de líquidos

Control del atrio izquierdo: catéter arterial pulmonar, con o sin monitorización y medición del rendimiento cardiaco.

Resucitación cardiopulmonar después de un paro durante las últimas 24 h (no considerar si se aplica únicamente golpe precordial).

\section{Asistencia renal}

16 Técnicas de hemofiltración, técnicas de diálisis.

17 Medición cuantitativa de orina (por ejemplo, mediante sonda vesical).

\section{Asistencia neurológica}

18 Medición de presión intracraneal.

\section{Asistencia metabólica}

19 Tratamiento de las complicaciones de acidosis/alcalosis metabólica.

20 Nutrición Parenteral Total Central.

Alimentación enteral mediante sonda gástrica u otros medios gastrointestinales (por ejemplo, yeyunostomía).

\section{Intervenciones específicas}

Intervenciones específicas en la unidad de cuidados intensivos: intubación endotraqueal, inserción de marcapasos, cardioversión, endoscopias, cirugías de emergencia durante las últimas 24 h,

22 lavado gástrico. Se excluyen las intervenciones rutinarias sin consecuencias directas a la condición clínica del paciente, tales como radiografías, ecografías, electrocardiogramas, curaciones o inserción de catéteres endovenosos o arteriales.

23 Intervenciones específicas fuera de la unidad de cuidados intensivos: cirugías o procedimientos de diagnóstico. 
tración de medicamentos ${ }^{(21)}$.

Lo mismo con el ítem 14 sobre el control del atrio izquierdo en donde se agregó, además de las mediciones, el término "monitorización”. Según los profesionales de enfermería, además de las mediciones hay una constante vigilancia e integración de las informaciones registradas en los monitores $y$, por lo tanto, una consecuente vigilancia de los pacientes, siendo esta actividad parte del control hemodinámico de los pacientes en el trabajo diario de las UCI en Chile.

Otro aspecto discutido en el grupo focal se refirió al profesional de kinesiología que ha asumido parcialmente el cuidado con las vías respiratorias. Los profesionales de enfermería estuvieron de acuerdo en mantener todos estos cuidados como "cuidados de enfermería" pues los kinesiólogos no los ejecutan en todo el período de 24 horas que contempla el instrumento, siendo estas actividades también ejecutadas por los profesionales de enfermería.

A su vez, los cuidados relacionados de higiene realizados por los técnicos de enfermería de nivel superior fueron asumidos como un cuidado complejo tratándose de pacientes críticos y, por tanto, la necesidad de un trabajo supervisado y colaborativo.

Otro punto discutido fue la innovación tecnológica y la actualización del instrumento. De lo anterior, un estudio multicéntrico en 19 UCI de siete países, liderado por el autor del instrumento original, describió la carga de trabajo de enfermería según el NAS y estableció una guía estandarizada para su utilización. La carga de trabajo de enfermería observada en las UCI de los siete países que participaron en el estudio mostró una gran variación del promedio NAS, siendo este hallazgo una información importante para la actualización y construcción de un manual de instrucciones del NAS estandarizado para su uso en la práctica clínica. Los autores concluyeron que la aplicación apropiada del NAS favorece la adopción de medidas reales que responden adecuadamente a la demanda del trabajo de enfermería y permite una mejor inversión en recursos humanos y materiales en la $\mathrm{UCI}^{(22)}$.

Cabe destacar que el instrumento adaptado transculturalmente favorecerá su utilización e irá en directo beneficio de la adopción de nuevos modelos de gestión de la dotación de personal de enfermería, derivado de la complejidad de los cuidados brindados a los pacientes y del tipo de servicios en la atención hospitalaria. Esto permitirá hacer una utilización eficiente del recurso humano y consecuente control de los costos, garantía de la calidad de los cuidados y satisfacción usuaria.

Evaluar a los pacientes con índices que midan objetivamente las intervenciones realizadas, así como también las necesidades de cuidados de enfermería pasan a ser una estrategia prioritaria en el contexto actual de la asistencia intensiva, frente a los costos involucrados en el sistema de salud.

En cuanto a la revisión del instrumento original una vez finalizada la adaptación, si bien la literatura recomienda esta etapa ${ }^{(23)}$, la misma no se realiza pues el instrumento ha sido ampliamente utilizado y documentado en su versión original.

\section{CONCLUSIÓN}

Si bien los jueces y enfermeros clínicos recomendaron ciertas modificaciones, estos obedecen a una necesaria adaptación transcultural de los ítems que, en general, fueron considerados bien diseñados y acordes para medir la carga de trabajo en enfermería en UCI en Chile.

\section{REFERENCIAS}

1. Queijo AF, Padilha KG. Nursing Activities Score (NAS): adaptación transcultural y validación para el portugués. Rev Esc Enferm USP. 2009; 43(Esp): 1018-25.

2. Tranquitelli AM, Padilha KG. Sistemas de classificação de pacientes como instrumentos de gestão em Unidades de Terapia Intensiva. Rev Esc Enferm USP. 2007; 41(1): 141-6.

3. Miranda DR, De Rijk A, Schaufeli W. Simplified Therapeutic Intervention Scoring Sys- 
tem: the TISS-28 items-results from a multicenter study. Crit Care Med. 1996; 24(1): 64-73.

4. Miranda DR, Nap R, De Rijk A, Schaufeli W, Iapichino G. The members of the TISS Working Group. Nursing activities score. Crit Care Med. 2003; 31(2): 374-82.

5. Palese A, Comisso I, Burra M, DiTaranto PP, Peressoni L, Mattiussi E, et al. Nursing Activity Score for estimating nursing care need in intensive care units: findings from a face and content validity study. J Nurs Manag. 2016; 24(4): 549-59.

6. Araújo TR, Menegueti MG, Auxiliadora-Martins M, Castilho V, Chaves LDP, Laus AM. Financial impact of nursing professionals staff required in an Intensive Care Unit. Rev Lat Am Enfermagem [Internet]. 2016 [cited 20 feb 2017]; 24: e2818. Available from: http://www.scielo.br/pdf/rlae/ v24/0104-1169-rlae-24-02818.pdf

7. Trettene AS, Luiz AG, Razera APR, Maximiano TO, Cintra FM, Monteiro LM. [Nursing workload in specialized Semi-intensive Therapy unit: workforce size criteria]. Rev Esc Enferm USP [Internet]. 2015 [cited $23 \mathrm{feb}$ 2017]; 49(6): 958-963. Available from : http:// www.scielo.br/pdf/reeusp/v49n6/0080-6234reeusp-49-06-0960.pdf

8. Armstrong E, De Waard MC, De Grooth HJ, Heymans MW, Reis Miranda D, Girbes AR, Spijkstra JJ. Using Nursing Activities Score to Assess Nursing Workload on a Medium Care Unit. Anesth Analg. 2015; 121(5): 1274-08.

9. Reich R, Vieira DF, Lima LB, Rabelo-Silva ER. [Nursing workload in a coronary unit according to the Nursing Activities Score]. Rev Gaucha Enferm [Internet]. 2015 [cited 02 mar 2017]; 36(3): 28-35. Available from: http://www.scielo.br/pdf/rgenf/v36n3/19831447-rgenf-36-03-00028.pdf

10. Valls-Matarín J, Salamero-Amorós M, Roldán-Gil C. [Analysis of the workload and the use of the nursing resources in an intensive care unit]. Enferm Intensiva. 2015; 26(2): 7281.

11. Oliveira LB, Rodrigues ARB, Püschel VAA,
Silva FA, Conceição SL, Béda LB et al. Assessment of workload in the postoperative period of cardiac surgery according to the Nursing Activities Score. Rev Esc Enferm USP. 2015; 49(spe): 80-86.

12. Nogueira LS, Padilha KG, Silva DV, Lança E de F, Oliveira EM, Sousa RM. Pattern of nursing interventions performed on trauma victims according to the Nursing Activities Score. Rev Esc Enferm USP. 2015; 49(Spec No): 29-35.

13. Silva JB, Póvoa VC, Lima MH, Oliveira HC, Padilha KG, Secoli SR. Nursing workload in hematopoietic stem cell transplantation: a cohort study. Rev Esc Enferm USP. 2015; 49(Spec No): 93-100.

14. Arias-Rivera S, Sánchez-Sánchez MM, Fraile-Gamo MP, Patiño-Freire S, Pinto-Rodríguez V, Conde-Alonso MP et al. Transcultural adaptation into Spanish of the Nursing Activities Score. Enferm Intensiva. 2013; 24(1): 12-22.

15. Alexandre NMC, Guirardello Ede B. [Cultural adaptation of instruments utilized in occupational health]. Rev Panam Salud Pública. 2002; 11: 109-111.

16. De Soárez PC, Kowalski CCG, Ferraz MB, Ciconelli RM. [Translation into Brazilian Portuguese and validation of the Work Limitations Questionnaire]. Rev Panam Salud Pública 2007; 22(1): 21-8.

17. Lynn M. Determination and quantification of content validity. Nursing Research. 1986; 35(6): 382-385.

18. Macedo AP, Mendes CM, Candeias AL, Sousa MP, Hoffmeister LV, Lage MI. Validação do Nursing Activities Score em unidades de cuidados intensivos portuguesas. Rev Bras Enferm. 2016; 69(5): 881-7.

19. Naveda OE, Naveda AF. Positive fluid balance and high mortality in paediatric patients with severe sepsis and septic shock. Pediatría. 2016; 49(3): 71-77.

20. González Pérez N, Zapata Centeno I, Gaona López R, Aguayo Muñoz A, Camacho Noguez A, López Carrillo L. Balance hídrico: un marcador pronóstico de la evolución clínica 
en pacientes críticamente enfermos. Reporte preliminar. Rev Asoc Mex Med Crit Ter Intensiva. 2015; 29(2): 70-84.

21. Toffoletto MC, Canales-Juan MA, Moreira-Arce D, Ordenes-Guerra A, Vergara-Rodríguez CA. Errors in the preparation and administration of medications: an integrative review of Latin American literature. Enferm. glob. 2015; 14(37): 350-360.

22. Padilha KG, Stafseth S, Solms D, Hoogendoom M, Monge FJC, Gomaa $\mathrm{OH}$, et al.
Nursing Activities Score: an updated guideline for its application in the Intensive Care Unit. Rev Esc Enferm USP. 2015; 49(Esp): 131-7.

23. Chávez LM, Canino G. Toolkit on Translating and Adapting Instruments. [Internet]. Human Services Research Institute; 2005 [citado 18 dic 2018]. 58 p. Disponible en: https://www.hsri.org/files/uploads/publications/PN54_Translating_and_Adapting.pdf 